\title{
International Comparisons of Femtosecond Laser Frequency Combs
}

\author{
Long-Sheng Ma, Zhiyi Bi, Albrecht Bartels, Lennart Robertsson, Massimo Zucco, Robert S. Windeler, \\ Guido Wilpers, Chris Oates, Leo Hollberg, and Scott A. Diddams
}

\begin{abstract}
Two types of international comparisons of femtosecond laser frequency combs have been performed in France and the USA. Five combs were involved in the comparisons. Three combs, of which two are transportable, employ nonlinear photonic crystal fiber (PCF) to obtain a wide spectrum covering a full optical octave. The other two are based on broadband femtosecond lasers and require no PCF. The comparisons were performed by counting the optical heterodyne beats between pairs of combs. The frequency agreement among three combs was at the subhertz level in the $563 \mathrm{THz}$ part of the comb spectrum when the combs were referenced to a hydrogen maser. When the combs were referenced to an optical standard, the frequency agreement among four combs was much improved and found to be at the $\sim 10^{-19}$ level in the spectral range of $333-473 \mathrm{THz}$. The fact that this result is obtained by five independent measurement systems (combs) strengthens the conclusion that no systematic effects are present at this level.
\end{abstract}

Index Terms-Femtosecond laser, frequency measurement, frequency standards.

\section{INTRODUCTION}

A BREAKTHROUGH in the applications of femtosecond laser (fs-laser) systems in frequency metrology was presented in 1999 [1], [2]. In a few years, such optical combs have become a crucial tool in the realization of the definition of the SI meter and a key component enabling one to take full advantage of the potential of future optical clocks based on a sharp optical resonance of a cooled and trapped ion, cooled atomic species or suitable molecules. In the development of future optical clocks, considering the high stability $\left(<10^{-15}\right.$ at $\left.1 \mathrm{~s}\right)$ and expected accuracy $\left(\sim 10^{-18}\right)$, a detailed study of the noise characteristics and possible systematic effects in the transfer between the optical and the radio frequency (RF) ranges is imperative if full

Manuscript received June 29, 2004. The work at the National Institute of Standards and Technology was supported in part by NASA. The project at East China Normal University was funded in part by the NSF of China (60490280, 10274020), STC(04JC14086, 04DZ14009), and SEC (2003-C007) of Shanghai.

L.-S. Ma is with the Bureau International des Poids et Mesures, Pavillon de Breteuil, 92312 Sèvres, France. He is also with the Physics Department, East China Normal University, Shanghai 200062, China (e-mail: lsma@phy.ecnu.edu.cn).

L. Robertsson is with the Bureau International des Poids et Mesures, Pavillon de Breteuil, 92312 Sèvres, France.

M. Zucco is with the Bureau International des Poids et Mesures, Pavillon de Breteuil, 92312 Sèvres, France, on leave from Consiglio Nazionale delle Ricerche-Istituto di Metrologia "Gustavo Colonnetti," 10135 Torino, Italy.

$\mathrm{Z}$. Bi is with the Physics Department, East China Normal University, Shanghai 200062, China.

A. Bartels, G. Wilpers, C. Oates, L. Hollberg, and S. A. Diddams are with the National Institute of Standards and Technology, Boulder, CO 80305 USA.

R. S. Windeler is with the OFS Laboratories, Murray Hill, NJ 07974 USA

Digital Object Identifier 10.1109/TIM.2005.843580 confidence in future standards is to be gained. As well as timekeeping, one of the most compelling motivations for the development of advanced optical frequency standards is that their comparison would allow one to search for possible time variations of fundamental constants [3], [4]. For example, laboratory based tests of the stability of the fine structure constant $\alpha$ show no time-dependence at the present measurement limit of $\sim 1 \times 10^{-15}$ per year [5], [6], while some astronomical measurements indicate that $\alpha$ could have been smaller than its present value by a factor of $1 \times 10^{-5}$ in the early universe some 10 billion years ago [7].

At the levels in question, well below existing standards, a direct comb-to-comb comparison appears to be the appropriate way to find the present limitations of this technique. To this end some comparisons and verification experiments have already been made [8]-[12]. Here we present the results of two international comparisons of fs-laser frequency combs, involving three laboratories and a total of five comb systems in two different designs. Such a comparison involving different systems not only tests the internal consistency in a comb spectrum but also sets limits on systematic effects, which could vary from system to system.

\section{EXPERIMENTS AND RESULTS}

Two international comparisons of fs-laser combs have been carried out at the Bureau International des Poids et Mesures (BIPM) in France and the National Institute of Standards and Technology (NIST) in the USA. Two transportable combs were first compared with the BIPM-C1 comb [13] at the BIPM. One of the transportable combs (BIPM-C2) was constructed by the BIPM and the other one (ECNU-C1) was constructed by the East China Normal University (ECNU) in Shanghai, China. These two combs have similar design and their configuration is shown in Fig. 1.

The two transportable combs are based on a femtosecond mode-locked Ti:sapphire laser that has a six-mirror ring cavity [14]. One of the mirrors is mounted on a fast piezo-electric transducer (PZT) that is used to control the repetition rate $f_{\text {rep. }}$ A second mirror is mounted on a long PZT, which is used to compensate the long-term drift of the fs-laser cavity. The fs-laser is pumped by a solid state laser at $532 \mathrm{~nm}$ with a pump power of $5 \mathrm{~W}$. The fs-laser pulses are first compressed by a pair of chirped multipass mirrors, before being coupled into a nonlinear PCF having a length of $30 \mathrm{~cm}$ [15]. The width of the fs-laser output spectrum is expanded to cover a full optical octave by the PCF. The mirror $M_{1}$ reflects the light near $1064 \mathrm{~nm}$, which is then frequency doubled to green light in a 5-mm-long 


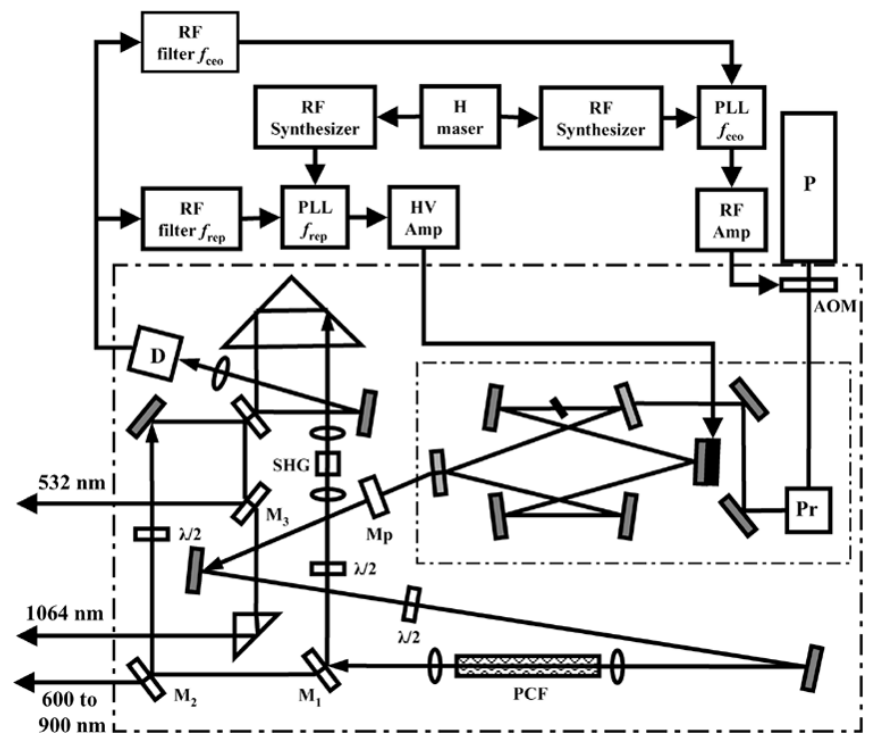

Fig. 1. Configuration of a transportable comb referenced to a hydrogen maser. (PLL: phase locked loop; HV Amp: driver of piezo-electric transducer; AOM: acoustooptic modulator; RF Amp: AOM driver; $\mathbf{M}_{1,2,3}$ : mirrors; $\mathbf{M p}$ : multipass chirped mirrors; P: pump laser; PCF: photonic-crystal-fiber; $\boldsymbol{\lambda} / 2$ : half waveplate; D: photo-detector; Pr: polarization rotator).

$\mathrm{KNbO}_{3}$ crystal. The mirror $M_{2}$ reflects the green beam near $532 \mathrm{~nm}$, which then is mixed with the frequency doubled light near $1064 \mathrm{~nm}$. This generates the beat signal for the control of the carrier envelope offset frequency $f_{\text {ceo }}$ at detector $\mathrm{D}$. The $f_{\text {ceo }}$ is phase locked to a RF synthesizer referenced to a hydrogen maser. The detector $\mathrm{D}$ is also used to obtain the servo signal for the control of the repetition rate $f_{\text {rep }}$, which also is phase locked to the same hydrogen maser by a second RF synthesizer. The fs-laser, PCF and self referencing setup are entirely contained in a sealed aluminum box with dimensions $69 \times 54 \times 23 \mathrm{~cm}^{3}$.

The transportable combs have three output beams each in different regions of the optical spectrum that allow measurements of three different optical frequencies simultaneously. The beam transmitted by $M_{2}$ passes through a window of the sealed box and has a total power of $30 \mathrm{~mW}$ in the spectral range $600-900 \mathrm{~nm}$. The beam transmitted by mirror $M_{3}$ is near $1064 \mathrm{~nm}$ and is steered through a second window of the sealed box by a right-angle prism. The total power of the output beam near $1064 \mathrm{~nm}$ is $20 \mathrm{~mW}$. The reflected light of mirror $M_{3}$ near $532 \mathrm{~nm}$ passes through a third window with a total power of $3 \mathrm{~mW}$.

The method used in the first international comparison of femtosecond laser combs at the BIPM has been described in detail in a previous report [16]. The repetition rates of the two transportable combs are near $800 \mathrm{MHz}$. We compared the two transportable combs with the BIPM-C1 comb, which has a repetition rate of $740 \mathrm{MHz}$. The combs were phase locked to a hydrogen maser by an independent phase locking system. An iodine stabilized Nd:YAG laser was employed as a common reference laser when comparing the two combs. The infrared beam of the iodine stabilized Nd:YAG laser was sent to the BIPM-C1 comb by a fiber to generate the beat signal $f_{\mathrm{b} 1}$ at $1064 \mathrm{~nm}$, which then was doubled to $2 f_{\mathrm{b} 1}$ by an RF doubler. The green beam of the iodine stabilized Nd:YAG laser was sent to the BIPM-C2 (or ECNU-C1) comb by a second fiber to generate the beat signal $f_{\mathrm{b} 2}$ at $532 \mathrm{~nm}$. The two beat signals were combined, and the summing part $\left(2 f_{\mathrm{b} 1}+f_{\mathrm{b} 2}\right)$ of the resultant signal was obtained by a double balanced mixer that links the optical beats between the two combs. The summed frequency $2 f_{\mathrm{b} 1}+f_{\mathrm{b} 2}$ is independent of the frequency drift of the Nd:YAG laser. The comparisons were performed by counting the beat signal of $2 f_{\mathrm{b} 1}+f_{\mathrm{b} 2}$ and comparing the resulting beat frequency with the expected value [16]. In the total measurement time of $18300 \mathrm{~s}$, the frequency agreement among the three combs (BIPM-C1, BIPM-C2, and ECNU-C1) was within $0.7 \mathrm{~Hz}$ at $563 \mathrm{THz}$. The precision of the measurement is limited by the noise of the RF synthesizers controlling $f_{\text {rep }}$.

In order to extend the measurements to include more systems and different techniques, the two transportable combs (BIPM-C2 and ECNU-C1) were shipped to NIST in Boulder and compared with the NIST broadband fiberless comb named NIST-BB1 [17], [18]. The NIST combs are based on four-mirror ring lasers that directly emit a broadband spectrum spanning the range $\sim 560-1150 \mathrm{~nm}$ at $-50 \mathrm{~dB}$ below the maximum. The total average output power is $640 \mathrm{~mW}$ with $8 \mathrm{~W}$ of pump light at $532 \mathrm{~nm}$. Additional broadening in a nonlinear PCF is therefore not required. Instead, the carrier envelope offset frequency can be measured by frequency tripling light emitted near $960 \mathrm{~nm}$ and heterodyning it with frequency doubled light near $640 \mathrm{~nm}$ [18], as shown in more detail in Fig. 2. The $f_{\text {ceo }}$ of each comb is phase-locked to a stable RF frequency synthesizer referenced to a hydrogen maser. The schematic of the comparison made at NIST is shown on Fig. 3. The beat signals, $f_{\mathrm{b} 1}$ and $f_{\mathrm{b} 2}$, between a continuous-wave $(\mathrm{CW})$ laser $(\lambda=657 \mathrm{~nm})$ and an individual element of each of the two combs, serve as servo signals for the phase locking to control the $f_{\text {rep }}$ of each comb relative to the CW laser frequency $f_{657}$, such that $f_{\mathrm{rep} 1,2}=\left(f_{657}-f_{\mathrm{ceo} 1,2}-f_{\mathrm{b} 1,2}\right) / N$, where $N$ is the mode order integer number.

Since the short term frequency stability of optical frequency standards can be one or two orders of magnitude better than that of hydrogen masers, the combs have much lower frequency noise when they are slaved to the cavity stabilized diode laser at $657 \mathrm{~nm}$ [19]. In the second comparisons, we used NIST-BB1 as a reference comb and compared this to the other three combs, BIPM-C2, ECNU-C1, and NIST-BB2 (a second broadband fiberless comb). Using two methods, four frequency combs were compared on six days over two months from August 15, 2003 to October 16, 2003 [20]. One of the methods involved the comparison of single lines adjacent to the control laser frequency $f_{657}$. This method does not require time synchronization between the optical pulse trains from the two frequency combs.

For the second method, the repetition rates of two frequency combs are required to be equal and the relative phase between the optical pulse trains from the two frequency combs is set to zero (i.e., the pulses from each frequency comb reach the detector at the same time). The optical heterodyne beat between two compared combs can be obtained by combining the two comb beams in a high speed photo diode. In this case a group of lines of the frequency combs generate a strong beat signal with a signal-to-noise ratio as high as $60 \mathrm{~dB}$ with a $300-\mathrm{kHz}$ bandwidth [21]. When the repetition rates of two combs are the same, 


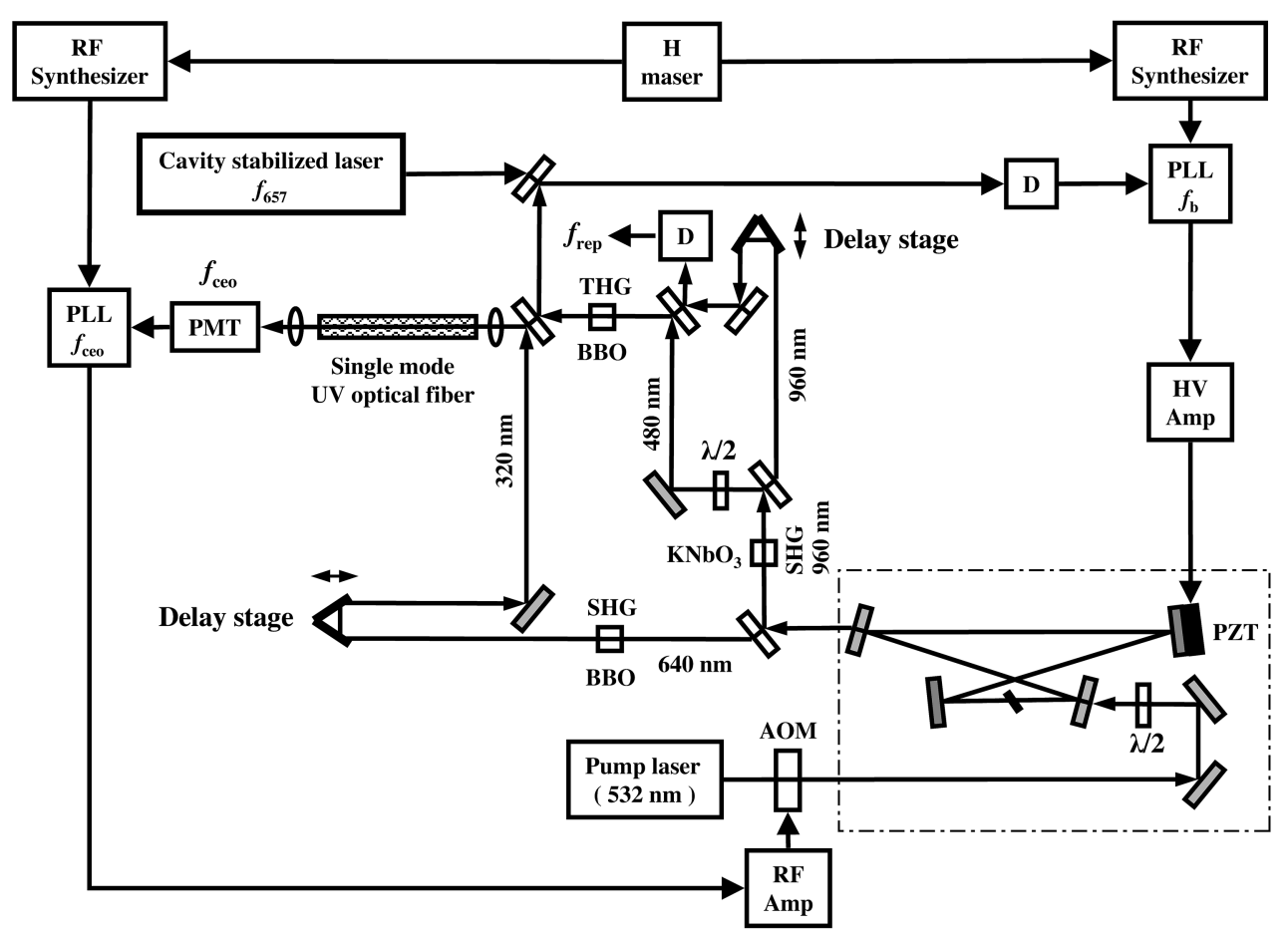

Fig. 2. Configuration of broadband combs of NIST-BB1 and NIST-BB2. (PZT: piezo-electric transducer; PLL: phase locked loop; HV Amp: PZT driver; AOM: acoustooptic modulator; RF Amp: AOM driver; $\boldsymbol{\lambda} / 2$ : half waveplate; $\boldsymbol{f}_{657}$ : frequency of cavity stabilized laser at $657 \mathrm{~nm} ; \mathbf{S H G}$ : second harmonic generation; THG: third harmonic generation; D: photo diode detector PMT: photomultiplier; $\boldsymbol{f}_{\text {ceo }}$ : carrier-envelop-offset frequency. $\boldsymbol{f}_{\text {rep }}:$ repetition rate; $\boldsymbol{f}_{\mathrm{b}}:$ beat frequency between $f_{657}$ and comb).

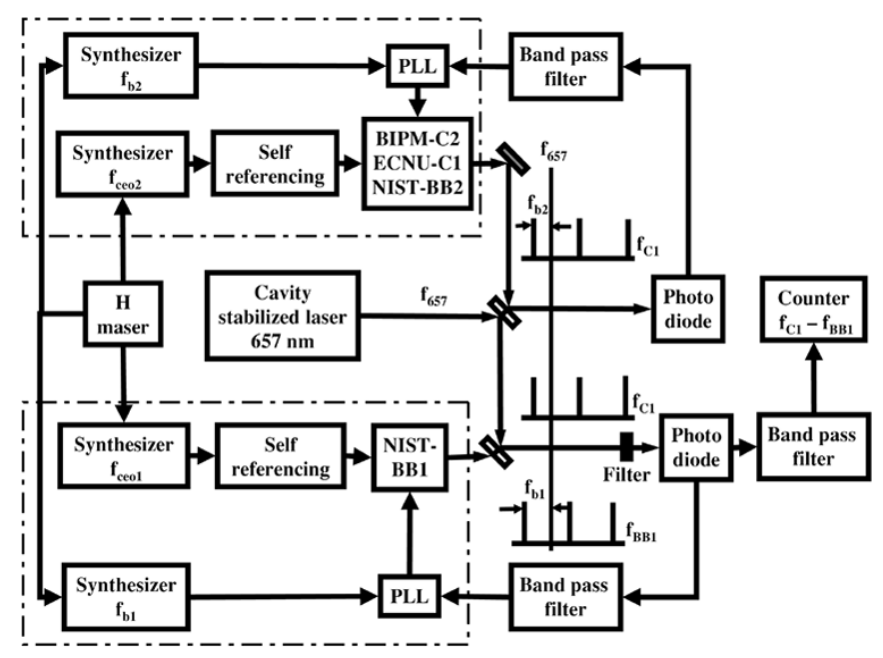

Fig. 3. Comparison of combs referenced to a cavity stabilized laser at 657 $\mathrm{nm}$. $\left(\mathbf{f}_{\mathrm{c} 1}\right.$ : frequency of BIPM-C2, ECNU-C1 and NIST-BB2 combs, $\mathbf{f}_{\mathrm{BB} 1}$ : frequency of NIST-BB1 comb).

the beat frequency is equal to $f_{\mathrm{ceo} 2}-f_{\mathrm{ceo} 1}$ and independent of the reference laser frequency $f_{657}[20]$. Any small difference of repetition rate between the two combs due to perturbation in phase control will be enhanced in the optical beat frequency by $N$ which is near $\sim 5 \times 10^{5}$. So measuring the optical beat frequency between compared combs is a very efficient way to test how precise the comb teeth are controlled by the reference laser at $f_{657}$. Interference filters were used to detect the beating signal between compared combs in different wavelength regions. The Doppler frequency shift due to thermal expansion of the experiment table and fluctuation of light paths both affect the stability of the beat frequency between the compared combs. In order to minimize such fluctuations of light path, we have arranged the optical paths for our experiments in a symmetric fashion and with as much common mode rejection as possible. Under such conditions and in the total measuring time of $51943 \mathrm{~s}$, the frequency agreement among the four compared combs was near $1 \times 10^{-19}$ (relative uncertainty with a confidence level of 95\%) [20].

After this series of measurements, the experimental environment conditions of the NIST combs were further improved. The two fs-laser frequency combs of NIST and all the measurement systems are now completely contained in two boxes, and the light beams transmitted between the combs are now protected by a tube. Moreover, the light path is also in more common mode. The fluctuation of the light path has in this way been made smaller and the short term instability of the beat frequency between two combs is at present improved by a factor of almost 10.

The two combs, NIST-BB1 and NIST-BB2, have recently been compared again, with the repetition rates of the two combs set to the same value. The comparisons were performed in the regions 456 and $473 \mathrm{THz}$. Under these improved conditions similar results as before, but with a shorter integration time (21060 s), were obtained. The average frequency difference between two combs was found to again be at the $10^{-19}$ level, essentially the same as the results obtained six months earlier.

\section{SUMMARY}

Two types of international comparisons of fs-laser combs have been performed using five combs of two different designs. 
TABLE I

The Results of Comparisons Using Five FemtoseCond LASER COMBS.

\begin{tabular}{c|c|c|c|c|c}
\hline $\begin{array}{c}\text { Compared } \\
\text { Combs }\end{array}$ & $\begin{array}{c}\text { Reference } \\
\text { Source }\end{array}$ & $\begin{array}{c}\text { Compared } \\
\text { Frequency }\end{array}$ & $\begin{array}{c}\text { Measurement } \\
\text { Time }\end{array}$ & $\begin{array}{c}\text { Relative } \\
\text { Uncertainty }\end{array}$ & $\begin{array}{c}\text { Measurement } \\
\text { period }\end{array}$ \\
\hline $\begin{array}{c}\text { BIPM-C1 } \\
\text { BIPM-C2, ECNU-C1 }\end{array}$ & $\begin{array}{c}\text { RF } \\
\text { Frequency }\end{array}$ & $563 \mathrm{THz}$ & $18300 \mathrm{~s}$ & $1.2 \times 10^{-15}$ & $\begin{array}{c}\text { Apr.5-Apr.9 } \\
2003\end{array}$ \\
\hline $\begin{array}{c}\text { NIST-BB1, NIST-BB2 } \\
\text { BIPM-C2, ECNU-C1 }\end{array}$ & $\begin{array}{c}\text { Optical } \\
\text { Frequency }\end{array}$ & $333-456 \mathrm{THz}$ & $51943 \mathrm{~s}$ & $1.4 \times 10^{-19}$ & $\begin{array}{c}\text { Aug.15-Oct.16 } \\
2003\end{array}$ \\
\hline $\begin{array}{c}\text { NIST-BB1 } \\
\text { NIST-BB2 }\end{array}$ & $\begin{array}{c}\text { Optical } \\
\text { Frequency }\end{array}$ & $\mathbf{4 5 6 - 4 7 3 \mathrm { THz }}$ & $\mathbf{2 1 0 6 0 \mathrm { s }}$ & $1.3 \times 10^{-19}$ & $\begin{array}{c}\text { Apr.1-Apr.21 } \\
2004\end{array}$ \\
\hline
\end{tabular}

The results of comparisons are listed in Table I. The frequency agreement among three combs, when referenced to a hydrogen maser, was at the subhertz level at $563 \mathrm{THz}$. When the combs are referenced to an optical standard, the comb frequency agreement among four combs was much improved and found to be at the $10^{-19}$ level in the spectral range of 333-473 THz. Considering that our experiments included five combs of different designs (broadband operation versus nonlinear PCF), it is significant that our data do not point to the existence of any fundamental limitations to the frequency reproducibility of the comb spectrum at the level of $\sim 10^{-19}$. Furthermore, the data were collected over a period of about nine months, in a long series of measurements and under changing conditions, which strengthens even more the confidence that no systematic effects exist at this level. The results demonstrate that fs-laser combs can indeed be a key element in future optical clocks and in searches for possible drift in fundamental constants.

\section{ACKNOWLEDGMENT}

The authors would like to thank T. Ramond, R. Fox, and J. Bergquist for their contribution to this work.

\section{REFERENCES}

[1] Th. Udem, J. Reichert, R. Holzwarth, and T. W. Hänsch, "Absolute optical frequency measurement of the cesium $D_{1}$ line with a mode-locked laser," Phys. Rev. Lett., vol. 82, pp. 3568-3571, 1999.

[2] D. J. Jones, S. A. Diddams, J. K. Ranka, R. S. Windeler, A. J. Stentz, J. L. Hall, and S. T. Cundiff, "Carrier-envelope phase control of femtosecond mode-locked lasers and direct optical frequency synthesis," Science, vol. 228, pp. 635-639, 2000.

[3] S. G. Karshenboim, "Some possibilities for laboratory searches for variations of fundamental constants," Can. J. Phys., vol. 78, pp. 639-678, 2000.

[4] J. D. Prestage, R. L. Tjoelker, and L. Maleki, "Atomic clocks and variations of the fine structure constant," Phys. Rev. Lett., vol. 74, pp. 3511-3514, 1995.

[5] H. Marion, F. Pereira Dos Santos, M. Abgrall, S. Zhang, Y. Sortais, S. Bize, I. Maksimovic, D. Calonico, J. Grünert, C. Mandache, P. Lemonde, G. Santarelli, Ph. Laurent, and A. Clairon, "Search for variations of fundamental constants using atomic fountain clocks," Phys. Rev. Lett., vol. 90, pp. 150801 1-150801 4, 2003.

[6] S. Bize, S. A. Diddams, U. Tanaka, C. E. Tanner, W. H. Oskay, R. E. Drullinger, T. E. Parker, T. P. Heavner, S. R. Jefferts, L. Hollberg, W. M. Itano, and J. C. Bergquist, "Testing the stability of fundamental constants with the ${ }^{199} \mathrm{Hg}^{+}$single-ion optical clock," Phys. Rev. Lett., vol. 90, pp. $1508021-1508024,2003$.
[7] J. K. Webb, M. T. Murphy, V. V. Flambaum, V. A. Dzuba, J. D. Barrow, C. W. Churchill, J. X. Prochaska, and A. M. Wolfe, "Further evidence for cosmological evolution of the fine structure constant," Phys. Rev. Lett., vol. 87, pp. 091301 1-091301 4, 2001.

[8] R. Holzwarth, Th. Udem, and T. W. Hänsch, "Optical frequency synthesizer for precision spectroscopy," Phys. Rev. Lett., vol. 85, pp. 2664-2267, 2000

[9] S. A. Diddams, L. Hollberg, L.-S. Ma, and L. Robertsson, "A femtosecond-laser based optical clockwork with instability $6.3 \times 10^{-16}$ in 1 s," Opt. Lett., vol. 27, pp. 58-60, 2000.

[10] J. Stenger, H. Schnatz, C. Tamm, and H. R. Telle, "Ultraprecise measurement of optical frequency ratios," Phys. Rev. Lett., pp. 88073601 1-88 $0736014,2002$.

[11] J. Ye, J.-L. Peng, R. J. Jones, K. W. Holman, J. L. Hall, D. J. Jones, S. A. Diddams, J. Kitching, S. Bize, J. C. Bergquist, L. W. Hollberg, L. Robertsson, and L.-S. Ma, "Delivery of high-stability optical and microwave frequency standards over an optical fiber network," J. Opt. Soc. Amer. B, vol. 20, no. 7, pp. 1459-1467, 2003.

[12] M. Zimmermann, Ch. Gohle, R. Holzwarth, Th. Udem, and T. W Hänsch, "Optical clockwork with an offset-free difference-frequency comb: Accuracy of sum- and difference-frequency generation," Opt. Lett., vol. 29, no. 3, pp. 310-312, 2004.

[13] L.-S. Ma, L. Robertsson, S. Picard, J.-M. Chartier, H. Karlsson, E Prieto, J. K. Ranka, and R. S. Windeler, "The BIPM laser standards at $633 \mathrm{~nm}$ and $532 \mathrm{~nm}$ simultaneously linked to the SI second using a femtosecond laser in an optical configuration," IEEE. Trans. Instrum. Meas., vol. 52, no. 2, pp. 232-235, Apr. 2003.

[14] A. Bartels, T. Dekorsy, and H. Kurz, "Femtosecond Ti:sapphire ring laser with a 2-GHz repetition rate and its application in time-resolved spectroscopy," Opt. Lett., vol. 24, pp. 996-998, 1999.

[15] J. K. Ranka, R. S. Windeler, and A. J. Stentz, "Visible continuum generation in air-silica microstructure optical fibers with anomalous dispersion at 800 nm," Opt. Lett., vol. 25, no. 1, pp. 25-27, 2000.

[16] L.-S. Ma, L. Robertsson, S. Picard, M. Zucco, Z. Bi, S. Wu, and R. S Windeler, "First international comparison of femtosecond laser combs at the International Bureau of Weights and Measures," Opt. Lett., vol. 29, no. 6, pp. 641-643, 2004.

[17] A. Bartels and H. Kurz, "Generation of a broadband continuum by a Ti:sapphire femtosecond oscillator with a $1-\mathrm{GHz}$ repetition rate," Opt. Lett., vol. 27, pp. 1839-1841, 2002.

[18] T. M. Ramond, S. A. Diddams, L. W. Hollberg, and A. Bartels, "Phase-coherent link from optical to microwave frequencies by means of the broadband continuum from a 1-GHz Ti:sapphire femtosecond oscillator," Opt. Lett., vol. 27, pp. 1842-1844, 2002.

[19] C. W. Oates, E. A. Curtis, and L. Hollberg, "Improved short-term stability of optical frequency standards: Approaching $1 \mathrm{~Hz}$ in $1 \mathrm{~s}$ with the Ca standard at 657 nm," Opt. Lett., vol. 25, pp. 1603-1605, 2000.

[20] L.-S. Ma, Z. Bi, A. Bartels, L. Robertsson, M. Zucco, R. S. Windeler, G. Wilpers, C. Oates, L. Hollberg, and S. A. Diddams, "Optical frequency synthesis and comparison with uncertainty at the $10^{-19}$ level," Science, vol. 303, pp. 1843-1845, 2004.

[21] R. K. Shelton, L.-S. Ma, H. C. Kapteyn, M. M. Murnane, J. L. Hall, and J. Ye, "Phase-coherent optical synthesis from separate femtosecond lasers," Science, vol. 293, pp. 1286-1288, 2001. 